\title{
Salicylaldehyde ester-mediated protein semi-synthesis enables studies on the tetra-acetylation of HMGB1
}

Tongyao Wei, ${ }^{[a]}$ Jiamei Liu, ${ }^{[a]}$ Yi Tan, ${ }^{[a]}$ Ruohan Wei, ${ }^{[a]}$ Jinzheng Wang, ${ }^{[a]}$ Hongxiang Wu, ${ }^{[a]}$ Yubo Tang, ${ }^{[\mathrm{a}]}$ and Xuechen $\mathrm{Li}^{*}{ }^{[\mathrm{a}]}$

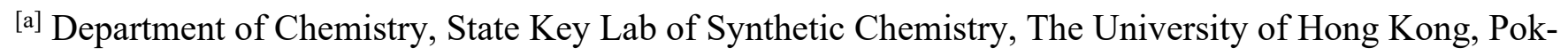
fulam Road, Hong Kong SAR (P. R. China)

KEYWORDS: Protein semi-synthesis $\bullet$ PTMs $・$ Chemical ligation $\bullet H M G B 1 ・$ Acetylation

\begin{abstract}
To answer how protein post-translational modifications (PTMs) affect protein function, conformation, stability, localization and interaction with binders remains important in the biological study. However, the related study has been dramatically hindered by the difficulty in obtaining homogenous proteins with site-specific PTMs of interest. Herein, we introduce a protein semi-synthesis strategy via salicylaldehyde ester-mediated chemical ligations (Ser/Thr ligation and Cys/Pen ligation). This methodology has enabled us to generate Lys (2/6/7/11) tetra-acetylated HMGB1 (high-mobility group box 1) protein, a $25 \mathrm{kDa}$ proinflammatory protein, in high purity. Further studies revealed that the tetra-acetylation may represent a regulatory switch to control the HMGB1 signaling pathway by abolishing its interaction with lipopolysaccharide (LPS) and accelerating its degradation, consequently preventing cells from pyroptosis and lethality upon infectious injury.
\end{abstract}

\section{INTRODUCTION}

Accessing proteins with desired natural or unnatural modifications is important yet challenging to correlate structure to function in the investigation of protein post-translational modifications (PTMs). ${ }^{[1,2]}$ Due to the non-template biogenesis of PTMs, biologically controlled methods cannot be used to produce homogeneous proteins bearing site-specific PTMs with precision and flexibility. Alternatively, advances in chemoselective peptide ligation chemistry enable peptide chemical synthesis to readily reach the protein domains comprising of 100-300 amino acid residues in general. ${ }^{[3-9]}$ In brief, side chain unprotected short peptides (less than 50 amino acids) obtained from solid phase peptide synthesis (SPPS) are chemoselectively merged specifically at $\mathrm{C}$ - and $\mathrm{N}$-termini via multiple chemical ligations. Along this line, the modified amino acids are used as the building blocks during SPPS with which to incorporate unnatural moieties into the polypeptide sequences, followed by protein refolding to generate functional synthetic biologics.

To maximize the efficiency of chemical synthesis of the proteins bearing PTMs, the semi-synthetic approach facilitated by chemical ligation offers an expedient solution, in which the short synthetic peptide 
containing modified residues is ligated with the long peptide fragment without PTMs obtained from recombinant expression. ${ }^{[10-19]}$ With the help of established techniques in recombinant protein production, the size of proteins accessed through semi-synthesis continues to increase in a cost-effective manner. ${ }^{[1,12]}$ Protein semi-synthesis lies in two manifolds: either C-terminal peptides or N-terminal peptides are biologically expressed. As peptide ligation relies on the reacting counterparts at C- and N-termini (e.g., Cterminal thioesters/N-terminal cysteine in native chemical ligation) for chemoselectivity, usage of natural amino acid as the reactive group would be advantageous for protein expression. For example, the protein with N-terminal cysteine for NCL can be readily obtained. In addition, a milestone advance has been made to generate recombinant proteins with $\mathrm{C}$-terminal thioesters via the intein splicing system, termed expressed protein ligation (EPL). ${ }^{[12]}$ However, the lowest abundance of cysteine among 20 natural amino acids limits the choice of ligation site, significantly impairing the applications of NCL in some cases. NCL-desulfurization method via $\mathrm{Cys} \rightarrow$ Ala conversion can expand the potential ligation junction to highly abundant alanine, yet it is only applicable to non-cysteine containing proteins or require temporal protecting group. ${ }^{[20-22]}$ Furthermore, proteins with other thiolated unnatural amino acids at N-terminus cannot be generated recombinantly yet. ${ }^{[23-25]}$

Previously, we have reported that unprotected peptides with N-terminal Ser/Thr can chemoselectively react with another unprotected peptides with $\mathrm{C}$-terminal salicylaldehyde esters to generate the N,O-benzylidene acetal linked intermediate in a pyridine/acetic acid mixture, which upon acidolysis restores the natural Xaa-Ser/Thr peptide linkage at the ligation site, termed Ser/Thr ligation (STL). ${ }^{[26,27]}$ In addition, the peptide salicylaldehyde esters can also react with peptides with N-terminal cysteine or penicillamine in both pyridine/acetic acid and aqueous buffers, termed Cys/Pen ligation (CPL), which is found to proceed independent of the steric hindrance at the C-terminal amino acid (e.g., Val, Ile. Thr and Pro). ${ }^{[28]}$ STL and CPL have been widely used for synthesis of cyclic peptides and proteins. ${ }^{[29-42]}$ As a further advance, STL/CPL-based protein semi-synthesis will be of great importance for large protein synthesis. The only example was reported by Kirshenbaum et al. in 2014, in which a peptoid derived salicylaldehyde ester was ligated with two proteins, RNase A $(\sim 11.5 \mathrm{kDa})$ and PTH (4 kDa) via STL. ${ }^{[43]}$ Since these two proteins are small and have a Ser at their N-terminus naturally, the development of a general protocol for STL and CPL in large protein semi-synthesis is needed. Herein, we report our systematic studies on exploring the application of STL and CPL in protein semi-synthesis and generating HMGB1 proteins with tetra-acetylation at lysine side chains of the $\mathrm{N}$-terminal region.

\section{RESULTS AND DISSCUSSION}

\section{Exemplify STL and CPL in large protein semi-synthesis.}

As the Ser/Thr ligation uses N-terminal Ser or Thr to mediate the peptide ligation, the protein with Nterminal Ser or Thr can be generated through proteolysis by several enzymes, as summarized in Figure S1. Furthermore, proteins with N-terminal Cys, used for CPL, can also by expressed in a similar manner (Figure 1A). Maltose-binding protein (MBP, $\sim 40 \mathrm{kDa}$ ) was chosen for the model study. MBP proteins with N-terminal Ser, Thr, or Cys were obtained by Ulp1 digestion. A number of typical peptide salicylaldehyde esters of different length were prepared via solid phase peptide synthesis (Figure 1B). After extensive condition screening (Table S1), the optimal conditions for STL are identified as following: MBP 
protein powder was dissolved in hexafluoro-2-propanol (HFIP) at $2 \mathrm{mM}$ and mixed with equal volume of pyridine/acetic acid solution $(1: 6, \mathrm{v} / \mathrm{v}) ; 10$ equivalents of peptide esters were added into the solution. Reaction was terminated by ether precipitation after 5 hrs. For CPL, MBP protein powder was dissolved in $6 \mathrm{M}$ Guanidine in the phosphate buffer $(\mathrm{pH} 4)$ at $1 \mathrm{mM}$ and 10 equivalents of peptide esters were added into the solution. Reaction was terminated by acetone precipitation after $12 \mathrm{hrs}$. The STL and CPL intermediates were then subjected to acidolysis with TFA/ $\mathrm{H}_{2} \mathrm{O} / \mathrm{EDT}(90 \% / 5 \% / 5 \%, \mathrm{v} / \mathrm{v} / \mathrm{v})$ for 5-30 minutes and precipitated by ether again. The final products were analyzed either by LC/MS or SDS-PAGE (Figure 1C, S2, S3, and S4). As shown in Figure 1C, MBP with N-terminal Thr always had the highest reactivity except for peptides with C-terminal Pro and Val salicylaldehyde (SAL) esters. MBP with N-terminal Cys could react with all the peptide SAL esters smoothly in $>60 \%$ conversion independent of the C-terminal amino acid. MBP with N-terminal Ser showed the lowest ligation efficiency but still could finish the reaction in two peptide esters (P1 and P2) with $>95 \%$ conversion based on LC/MS. According to these results, we believed that under the optimized condition, the STL/CPL on large protein semi-synthesis and on short peptides synthesis share the same reaction features (Table S2).
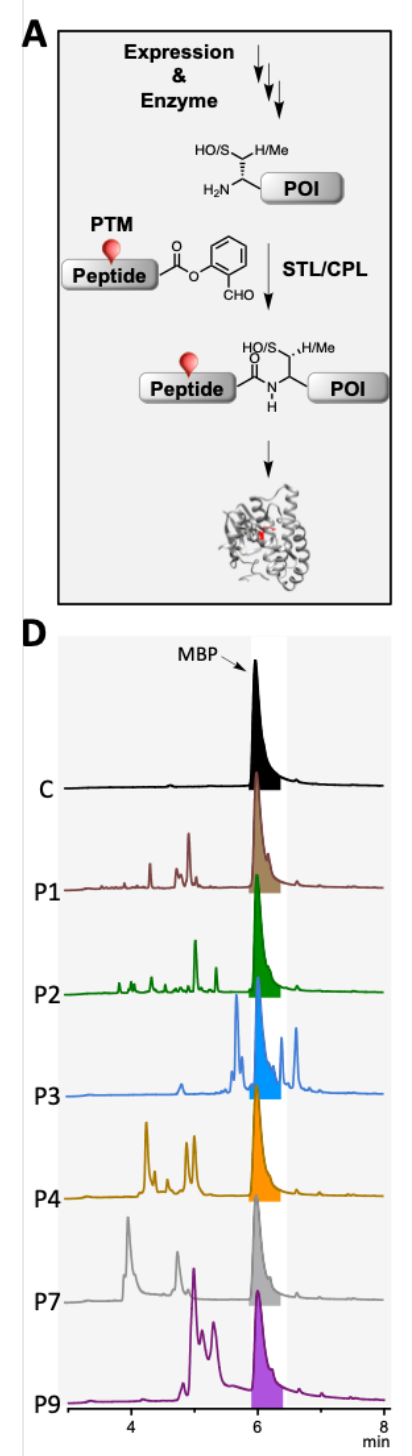
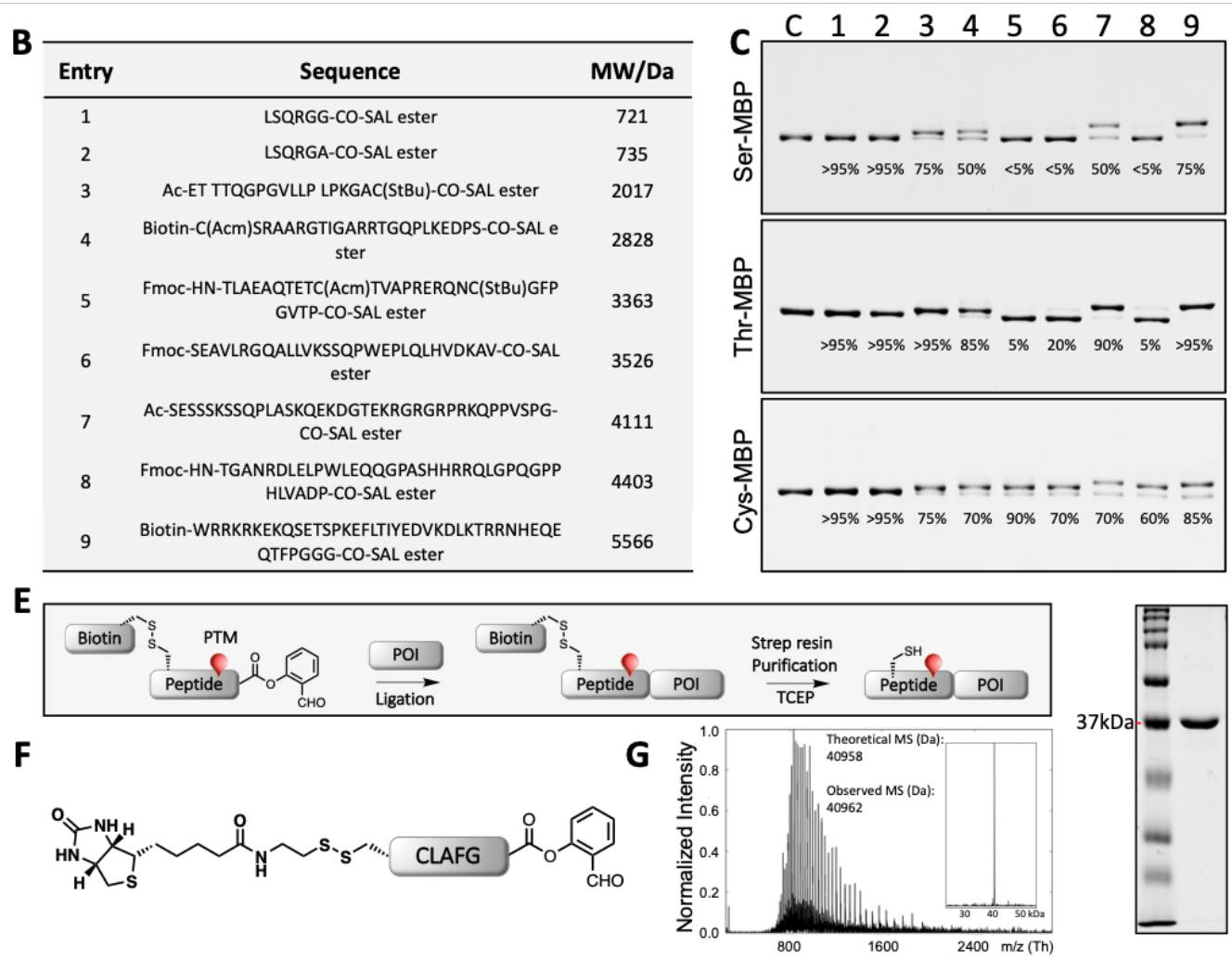

H

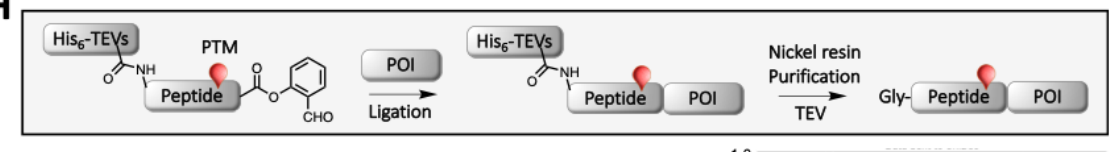

I
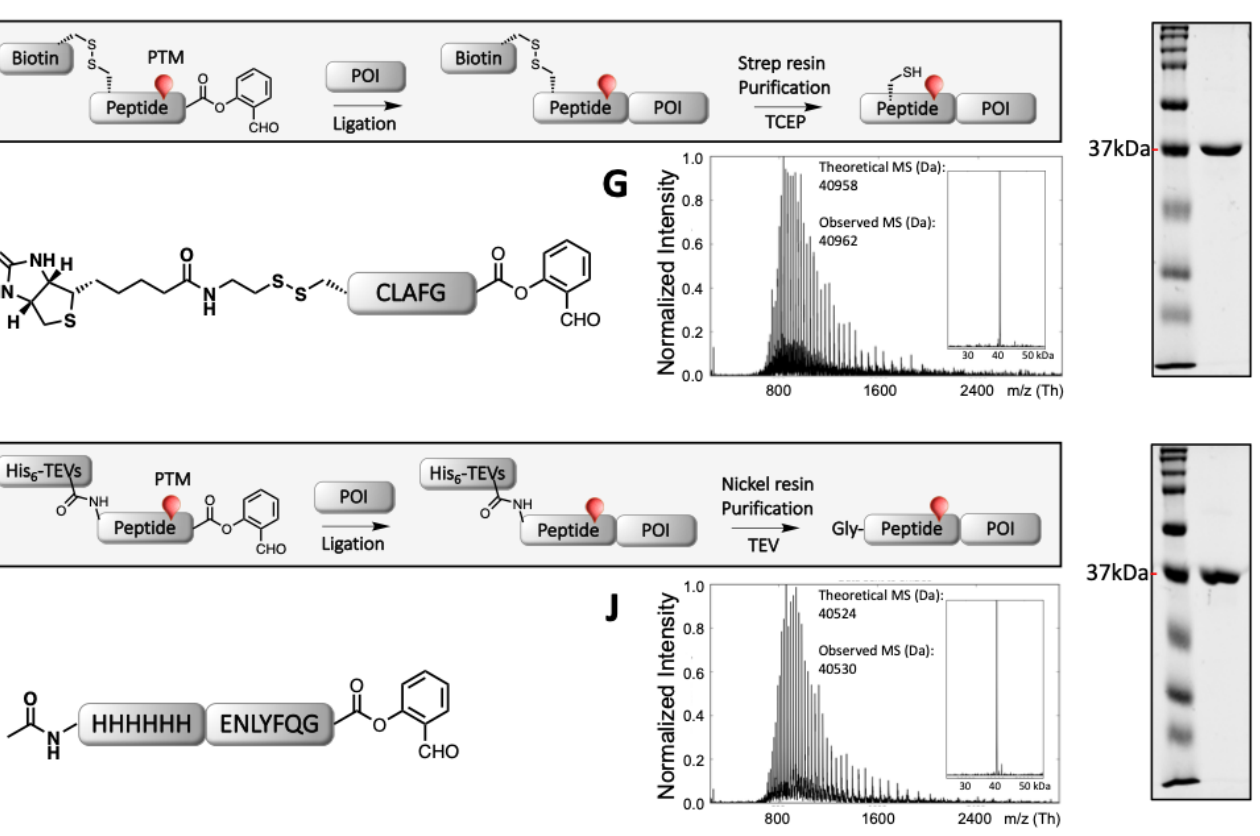
Figure 1. STL/CPL mediated protein semi-synthesis and Purification strategies.A. Overview of STL/CPL mediated protein semi-synthesis. B. Peptide esters used for demonstrating the ligation. SAL: Salicylaldehyde. C. SDS-PAGE analysis of ligation products. Conversion were calculated according the peaks intensity after deconvolution and indicated below the bands. See also Figure S2, S3, and S4. c: control. D. UV traces of reaction mixtures of the indicated peptide esters reacting with Ser-MBP. The reactions with more than 50\% conversion were chosen (Figure $1 \mathrm{C}$ top). No shift was observed on the HPLC UV trace after ligation. Except for the product, other peaks mainly were the unconsumed peptide ester, the hydro-lysed peptide ester and the product of salicylaldehyde esters being displaced by HFIP. E. Disulfide linker-mediated purification strategy. Strep resin: streptavidin resin. F. The sequence of model peptide for disulfate linker-mediated purification strategy. G. Left: Mass spectrum of the final products; Right: SDS-PAGE analysis of the final product. H. His tag and TEV based purification strategy. TEVs: TEV recognition site. I. The sequence of model peptide for His tag and TEV based purification strategy. Note: this ligation can be fully converted after $3 \mathrm{~h}$. In order to demonstrate our design, we stopped the reaction after $30 \mathrm{~min}$. J. Mass spectrum of the final product; Right: SDS-PAGE analysis of the final product. See also Figure S5.

As there is a minimal effect on the polarity of the large protein after it is ligated with a short peptide, the final product often overlapped with the unconsumed expressed peptide part on reversed-phase HPLC, which made the purification difficult (Figure 1D). To facilitate protein purification, we introduced an extra tag, either His 6 or biotin into the synthetic peptide fragment for subsequent purification. To this end, we used the traceless disulfide linker, which is applicable in cysteine-containing protein. The tag could be removed by TCEP treatment after purification (Figure 1E, F, and G). Alternatively, the enzymatically cleavable sequence was used, such as TEV recognition site (Figure 1H, I, and J). After purification, the tag could be removed by TEV digestion, leaving an extra Gly at the N-terminus of the protein. This strategy turned out to be very effective to generate homogeneous synthetic proteins, as shown in Figure 1J.

\section{Semi-synthesis of HMGB1 with tetra-acetylation.}

To further demonstrate the efficiency of our methodology, the high-mobility group box 1 (HMGB1) protein with important activity in inflammation process, was chosen as the synthetic target. HMGB1 as a mammalian nuclear protein can translocate to cytoplasm and outside of cells eventually during bacterial infection or sterile tissue injury. ${ }^{[43]}$ Extracellular HMGB1 is one of damage-associated molecular pattern (DAMP) proteins that acts as a proinflammatory molecule, facilitating migration and activation of inflammatory cells through Toll-like receptor 4 (TLR4) and advanced glycation end products (RAGE). ${ }^{[45]}$ It is worth mentioning that the relevance of extracellular HMGB1 as a biomarker or therapeutic target has been demonstrated in many diseases. ${ }^{[46,47]}$ For instance, a deleterious systemic inflammatory response (endotoxemia and bacterial sepsis) can be evoked by the circulating bacteria, while it can be subsided by neutralizing extracellular HMGB1, subsequently preventing caspase-11-dependent pyroptosis and death. ${ }^{[48]}$ 

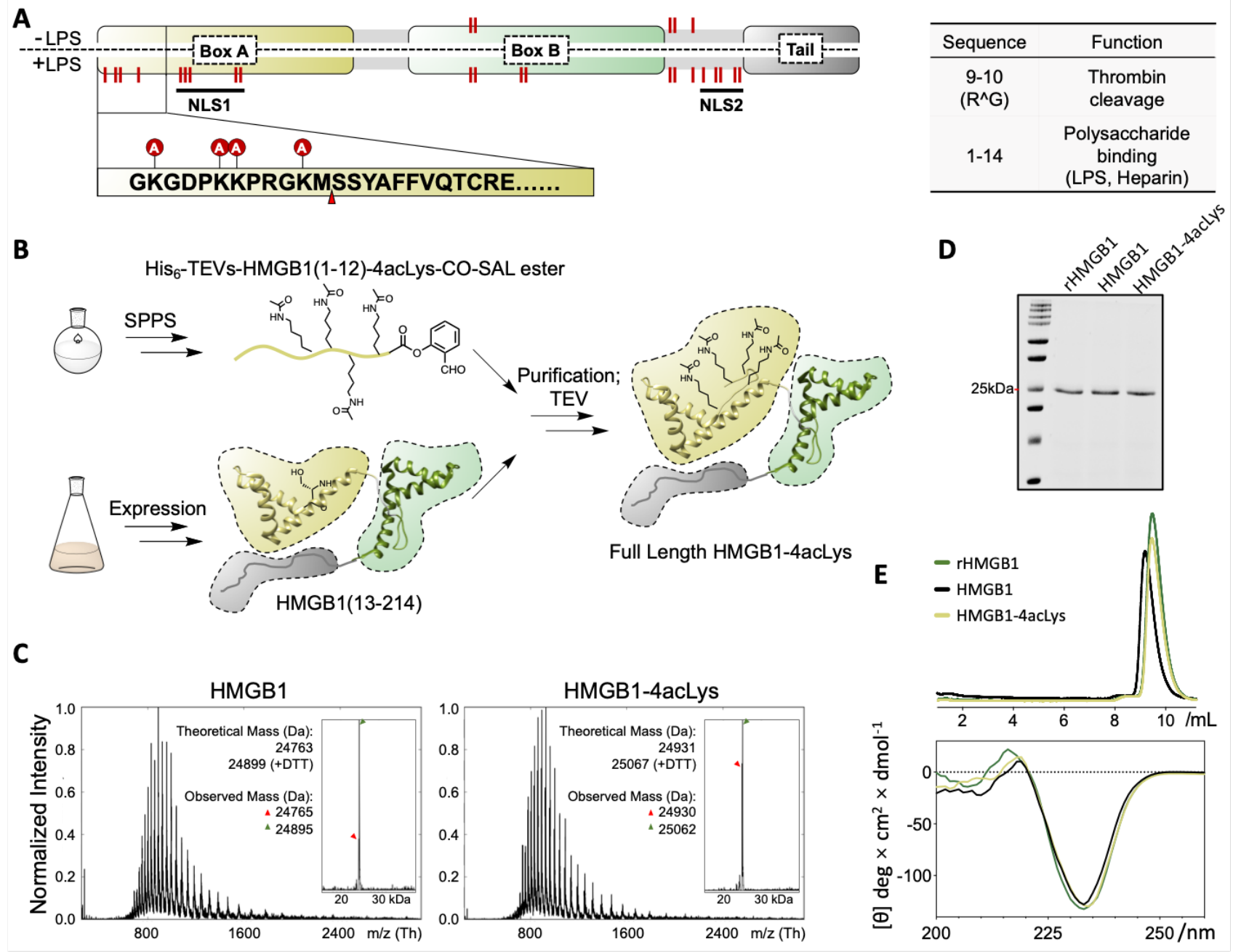

Figure 2. Tetra-acetylated HMGB1 (HMGB1-4acLys) and wild type HMGB1 (HMGB1) were chemical synthesized. A. HMGB1 was acetylated after LPS stimulation. The modification sites were highlighted by red lines. Right: The N-terminus mediated functions. The ligation site was indicated by an arrowhead. B. Chemical synthesis routes of HMGB1. C. Mass spectra of the synthetic HMGB1 and HMGB1-4acLys. Note: Intein tag-based purification will install a DTT ester on the C-terminus of protein, see also Figure S6. D. SDS-PAGE analysis of synthetic HMGB1proteins. Recombinant full length HMGB1 (rHMGB1) was purified under native condition and was loaded as the control. E. Refolding of synthetic HMGB1 proteins. Up: The FPLC spectra showed the HMGB1 proteins were well folded as the monomer; Bottom: The circular dichroism (CD) spectra of HMGB1 proteins showed similar pattern.

Efficient secretion of HMGB1 requires hyper acetylation on its two nuclear localization sequences (NLS) which afterwards causes the accumulation in the cytoplasm. Previous studies have shown that in addition to the NLS sites, significant acetylation also was found at the unstructured N-terminal region (Lys2, 6, 7, and 11) when cells were exposed to lipopolysaccharide (LPS, one of pathogen-associated molecular patterns to stimulate HMGB1 release) (Figure 2A) ${ }^{[49]}$ Interestingly, another study showed that extracellular HMGB1 could interact with LPS through its N-terminus (selectively interact with the polysaccharide (PS) moiety of LPS) and the linker between box A and box B (selectively interact with the lipid A moiety of LPS) of HMGB1, promoting LPS internalization through RAGE for cytosolic LPS receptor to trigger more serious inflammatory responses, for example, cell pyroptosis and lethality. ${ }^{[50,51]}$ 
That is, for HMGB1, the LPS-stimulated acetylation remarkably occurs at the binding site of LPS. However, whether the acetylation affects the HMGB1-LPS interaction remains largely unexplored.

To study the effect of the tetra-acetylation at Lys2, 6, 7, and 11, we set out to chemically synthesize HMGB1 with the tetra- acetylation. Of note, there is a Cys22 residue for performing NCL, however the ligation at Thr21 presents a difficult site. ${ }^{[11]}$ Also, the unnatural amino acid incorporation technique may produce the protein with acetylation at one single lysine residue by fully recombinant approach. ${ }^{[52]}$ Here, we mainly focus on the protein semi-synthesis using Ser/Thr ligation strategy, which uses a short synthetic peptide fragment (12aa with a removable tag) (Figure 2B). The synthesis of HMGB1 was designed to disconnect at Met12-Ser13 via Ser ligation. To this end, HMGB1(13-214) was expressed via TEVmediated proteolysis, while the peptide (1-12) salicylaldehyde ester with His6-TEV cleavage site and Lys acetylation was chemically synthesized. The ligation proceeded smoothly with $35 \%$ conversion after 5 hrs according to the deconvoluted peak intensity (the other $65 \%$ remain as starting material). After nickel resin purification, TEV cleavage, refolding, and FPLC purification, the full-length HMGB1 with lysine acetylation was obtained with 10\% overall isolated yield (Figure 2C, and D) and well folded (Figure 2E).

\section{Tetra-acetylation inhibits HMGB1-lipopolysaccharide interaction.}

To investigate whether the acetylation regulates the HMGB1-polysaccharide interaction, we used the biotinylated polysaccharide of LPS to pull-down HMGB1 in vitro. As shown in Figure 3A, S7, the Nterminus deleted HMGB1 lost the binding with the polysaccharide compared with the full length HMGB1. Importantly, tetra-acetylation also significantly impaired the HMGB1-polysaccharide interaction. Moreover, the intact biotinylated LPS pull-down experiment revealed that the interaction between N-terminus of HMGB1 and the polysaccharide is necessary for the formation of HMGB1-LPS complex, which can be regulated by the tetra-acetylation, suggesting the potentially vital effect of the tetra-acetylation on the LPS-mediated inflammatory response.
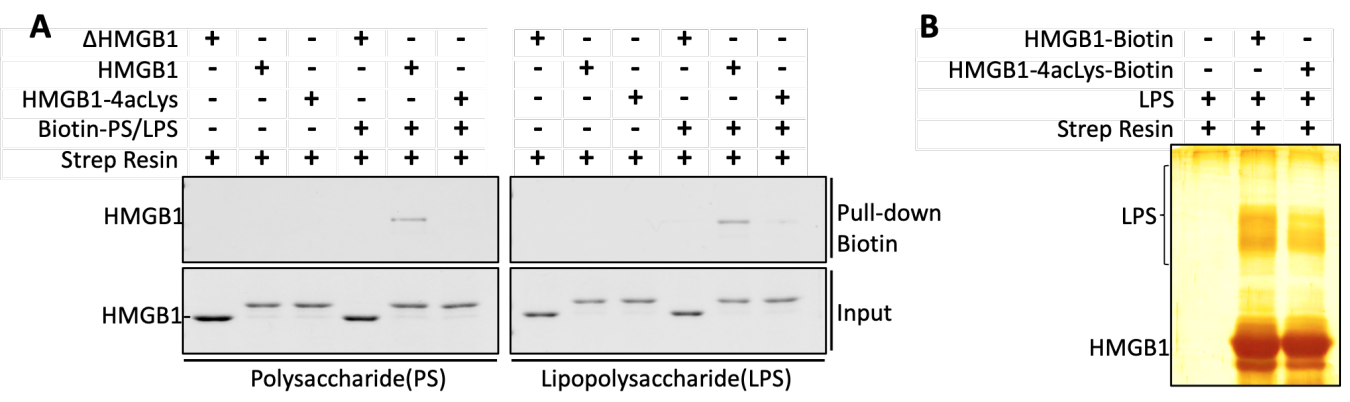

Figure 3. Tetra-acetylation is involved in the LPS binding. A. Pull-down HMGB1 proteins using biotin-polysaccharide (PS) or biotin-lipopolysaccharide (LPS). $\mathrm{HMGB1}$ : HMGB1 (13-214, N-terminus deleted) was purified under native condition. B. Pull-down LPS using biotin-HMGB1 proteins. LPS was extracted from E. coli. BL21. See also Figure S7, S8.

To further validate the result, the full length HMGB1 with tetra-acetylation at its N-terminus and a biotin tag at its C-terminus was generated via combining STL and NCL (Figure S8). The compatibility of STL and NCL enabled us to chemically modify HMGB1 at two terminus without complicated procedure. ${ }^{[10]}$ Pull-down experiment clearly showed that the acetylation indeed hindered the binding of LPS to 
HMGB1, suggesting an important mechanism at the posttranslational level, which may effectively regulate the proinflammatory function of HMGB1 (Figure 3B).

\section{Tetra-acetylation accelerates the thrombin-mediated degradation.}

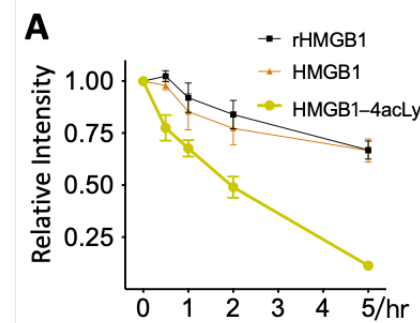

C

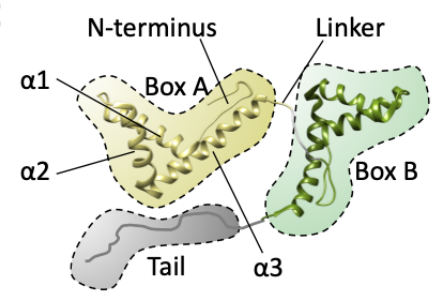

D
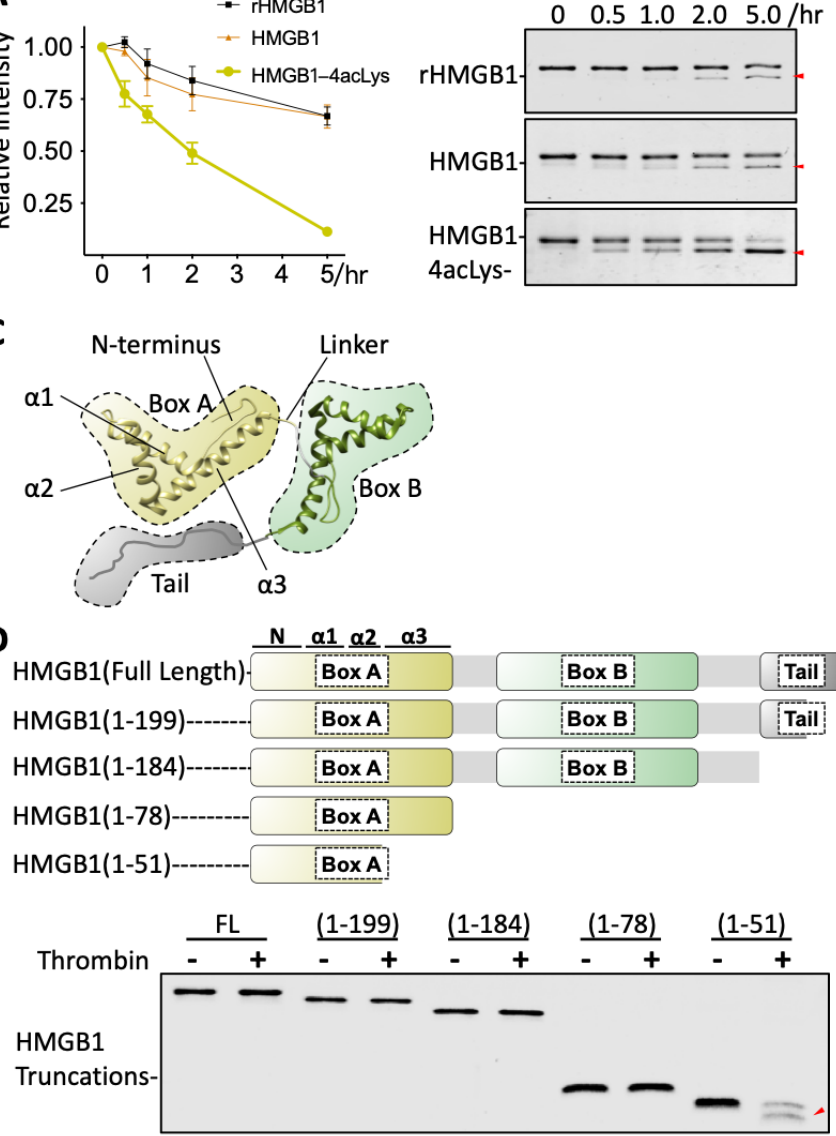

\section{B}
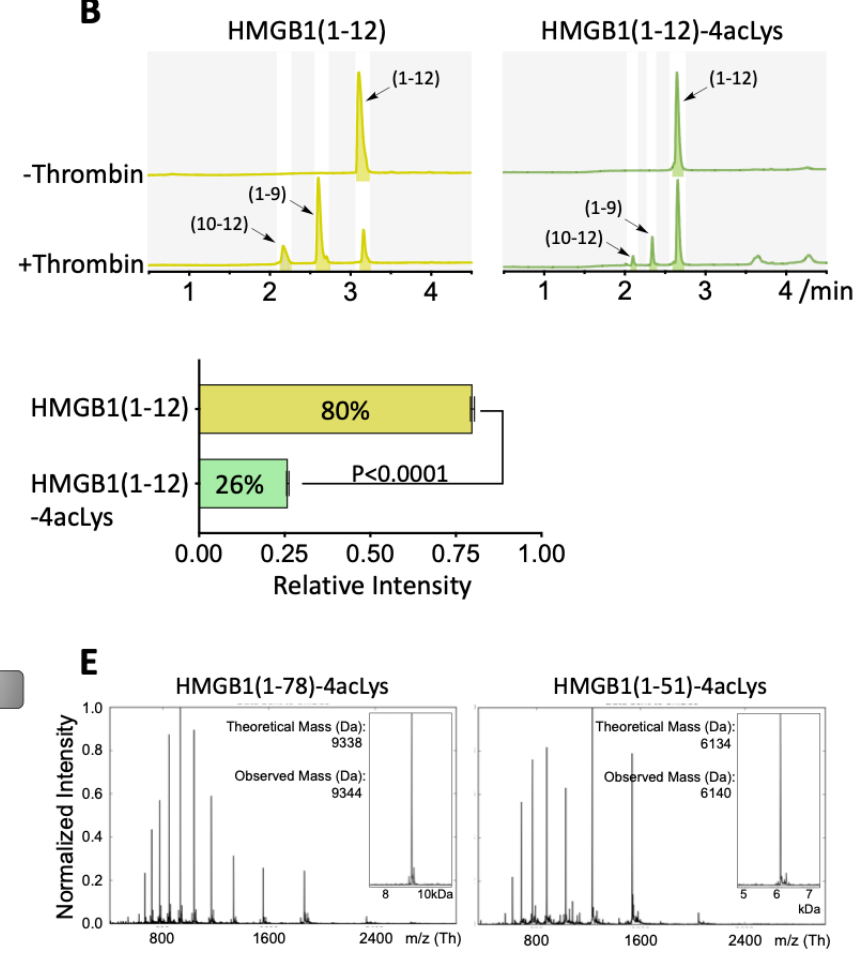

(1-78)-

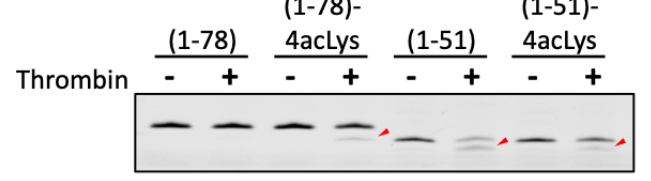

Figure 4. Tetra-acetylation is involved in thrombin-mediated degradation. A. HMGB1 digestion assay. Left: Bands were quantified to generate the digestion rate curve. Error bars indicate average $\pm S E s, n=3$. Right: Representative SDS-PAGE analysis. B. Thrombin digestion assay on HMGB1(1-12) peptide. Left: UV traces of the digested HMGB1(1-12) mixture after treating with thrombin for $0.5 \mathrm{~h}$. Right: UV traces of the digested HMGB1(1-12)4acLys mixture after treating with thrombin for $0.5 \mathrm{~h}$. Bottom: The rate of the consumed material vs the starting material was calculated by integrating the UV peaks of HMGB1(1-12). Error bars indicate average \pm SEs, $n=3$. C. Overview of HMGB1's structure. $\alpha 1$ : the first $\alpha$-helix; $\alpha 2$ : the second $\alpha$-helix; $\alpha 3$ : the third $\alpha$-helix. PDB: 2 YRQ. D. Up: HMGB1 truncations in this study. Bottom: SDS-PAGE analysis of digested HMGB1 truncations at $0.5 \mathrm{~h}$. E. Up: Mass Spectra of semi-synthetic HMGB1(1-78)-4acLys and HMGB1(1-78)-4acLys; Bottom: SDS-PAGE analysis of digested HMGB1(1-78), HMGB1(1-78)-4acLys, HMGB1(1-51), HMGB1(1-51)-4acLys at $0.5 \mathrm{~h}$.

In addition, a serine protease in vascular endothelium, thrombin, could recognize and cleave the Nterminus, producing a less proinflammatory form of HMGB1. ${ }^{[53]}$ To evaluate the effects of the tetra-acetylation on the HMGB1 degradation, a thrombin digestion assay was performed. As shown in Figure 4A, the thrombin had comparable proteolysis activity on the expressed full length HMGB1 and the synthetic HMGB1-4Lys. Unexpectedly, the tetra-acetylation considerably enhanced thrombin-mediated cleavage of HMGB1. 
According to a previous study, the aliphatic residues (e.g. Leu and Gly) at P4 was preferred by thrombin (Figure S9). ${ }^{[54-56]}$ As the acetylation on Lys6 (P4) could dramatically decrease the hydrophilicity of Lys through installing an acetyl group on their side chains, the acetylated HMGB1 will likely become a more optimal substrate for thrombin. To verify our hypothesis, the HMGB1(1-12) and HMGB1(1-12)-4acLys peptides were synthesized. Nevertheless, the HMGB1 digestion assay has shown that thrombin could cleave HMGB1(1-12) more effectively than HMGB1(1-12)-4acLys (Figure 4B). These conflict results promoted us to deduce that the conformational hindrance may be the key point to affect the enzyme activity, even though the CD spectra did not show obvious difference between the wild type and the tetraacetylated HMGB1. In this case, the unacetylated N-terminus of HMGB1 interacts with other regions of HMGB1, while acetylation abolishes this interaction and releases the $\mathrm{N}$-terminus for thrombin recognition and cleavage. Therefore, we generated several HMGB1 truncations recombinantly and performed the digestion assay (Figure 4C, and D). Although previous studies have proved that the acidic tail interacts with the box $A,{ }^{[57]}$ our results did not show significant improvement of thrombin activity until truncating HMGB1 from the third $\alpha$-helix ( $\alpha 3)$, which indicated that the N-terminus of HMGB1 interacts with the $\alpha 3$ tightly. In addition, we also synthesized the HMGB1(1-78) and HMGB1(1-51) with tetra-acetylation. Thrombin digestion assay clearly showed that like N-terminal peptides, the enzyme activity on HMGB1(1-51) is higher than the tetra-acetylated form (Figure 4E), proving that the acetylation indeed impairs the interaction between the $\mathrm{N}$-terminus and the third $\alpha$-helix, thereby may disrupting the entire Box A structure through this way. In summary, tetra-acetylation could liberate the N-terminus from the intramolecular interaction, and thrombin becomes to be more effective to cleave and inactivate the HMGB1, consequently may sequester the inflammatory response upon infectious injury.

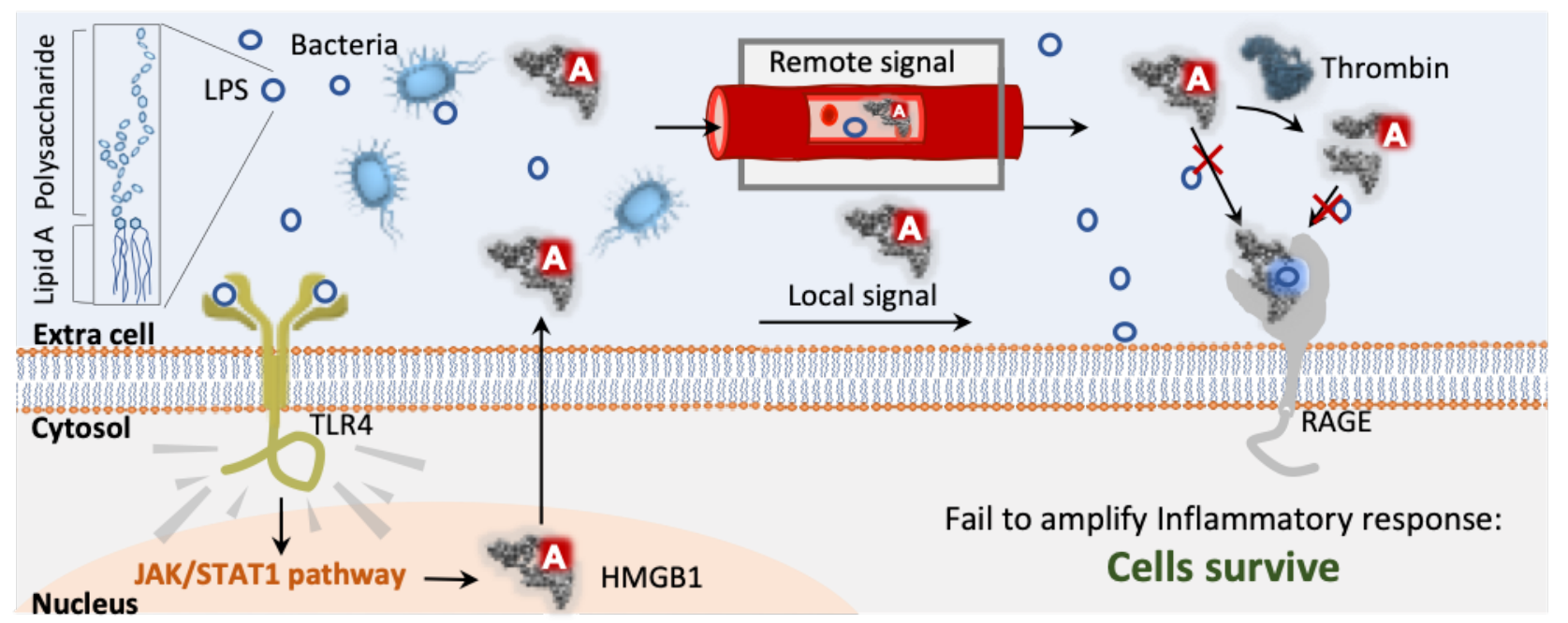

Figure 5. Proposed outcoming for tetra-acetylation of HMGB1. LPS, which is released from circulating bacteria, binds to TLR4, and stimulates JAK/STAT1 signaling pathway. Afterwards, HMGB1 is hyper acetylated including the tetra-acetylation at the N-terminus and secreted. However, the tetra-acetylated HMGB1 is unable to bind LPS, and degraded rapidly by thrombin. As a result, the tetra-acetylated HMGB1 fails to stimulate more severe inflammatory responses, and cells survive.

\section{CONCLUSION}


Notably, the main key to explore how the PTMs contribute to the multifarious cellular activities at a molecular level increasingly relies on our ability to access the homogenous proteins with site-specific PTMs of interest. Chemical synthesis offers a useful tool to generate proteins with customized modifications (e.g. PTMs). Thus, a variety of different proteins with complicated modifications (e.g. glycosylation) are becoming accessible. ${ }^{[58,59]}$ Nonetheless, the advantage of chemical protein synthesis still remains unfulfilled since the relatively burdensome total synthesis is difficult to start at a biological laboratory. A1ternatively, chemical ligation enabled protein semi-synthesis, which combines the strengths of organic synthesis and recombinant DNA technology, is more feasible for biologists, as small synthetic peptide parts will be affordable or synthetically accessible by themselves.

In this study, we have delved into the application of STL and CPL in protein semi-synthesis. The success of stitching different peptide salicylaldehyde esters with the N-terminus of MBP, a $40 \mathrm{kDa}$-protein, exemplified the operability of STL and CPL on large protein synthesis. Furthermore, two purification strategies were introduced for separating the ligated product from the unreacted or hydrolyzed starting materials without using HPLC purification. Finally, using this strategy, we generated the full-length tetraacetylated HMGB1 with high purity, which enabled us to illustrate that the N-terminal tetra-acetylation may represent a regulatory switch to control the HMGB1's signal pathway and stability. We could conclude that the acetylation may subverts the proinflammatory function of HMGB1 by abolishing interaction with LPS and accelerating the degradation, which consequently serves as a rescue mechanism from pyroptosis and lethality for cell upon infectious injury (Figure 5). In addition, the critical interaction between the HMGB1 N-terminus and polysaccharide (e.g. LPS and heparin) probably has underpinned the development of sugar-based inhibitors for HMGB1, which is the most popular strategy nowadays. ${ }^{[45]}$ Thus, a detailed study on the N-terminus modification will largely benefit the development of HMGB1related drug. In addition, as this study mainly focused on the overall influence of tetra-acetylation on the function and stability of HMGB1, these acetylation sites may need to be further investigated individually.

In summary, STL/CPL-mediated protein semi-synthesis can serve as a good tool for facilitating the solution to the inaccessible protein (with PTMs), unveiling more information at a molecular level.

\section{ASSOCIATED CONTENT}

\section{Supporting Information}

Supplementary figures, table and synthetic details (PDF)

\section{AUTHOR INFORMATION}

\section{Corresponding Author}

* Xuechen Li: xuechenl@hku.hk

\section{Author Contributions}

All authors have given approval to the final version of the manuscript.

\section{Funding Sources}


The work was supported by the Grants Research Council of University Grants Committee of Hong Kong (C7147-20G, 17303920, AoE/P-706/16).

\section{ACKNOWLEDGMENT}

We thank Dr. Han Liu for proofreading the manuscript. We thank Miss. Zirong Huang for synthesis efforts.

\section{REFERENCES}

1. Liu, L. Chemical synthesis of proteins that cannot be obtained recombinantly. Isr. J. Chem. 2019, 59, 64-70.

2. Tan, Y.; Li, J.; Jin, K.; Liu, J.; Chen, Z.; Yang, J.; Li, X. Cysteine/Penicillamine Ligation Independent of Terminal Steric Demands for Chemical Protein Synthesis. Angew. Chem. Int. Ed. 2020, $132,12841-12845$.

3. Schnolzer, M.; Kent, S.B. Constructing proteins by dovetailing unprotected synthetic peptides: backbone-engineered HIV protease. Science 1992, 256, 221-225.

4. Dawson, P.E.; Muir, T.W.; Clark-Lewis, I.; Kent, S.B. Synthesis of proteins by native chemical ligation. Science 1994, 266, 776-779.

5. Albericio, F.; Lloyd-Williams, P.; Giralt, E. Convergent solid-phase peptide synthesis. Methods Enzymol. 1997, 289, 313-336.

6. Bondalapati, S.; Jbara, M.; Brik, A. Expanding the chemical toolbox for the synthesis of large and uniquely modified proteins. Nat. Chem. 2016, 8, 407-418.

7. Agouridas, V.; El Mahdi, O.; Diemer, V.; Cargoët, M.; Monbaliu, J.C.M.; Melnyk, O. Native chemical ligation and extended methods: mechanisms, catalysis, scope, and limitations. Chem. Rev. 2019, 119, 7328-7443.

8. Bode, J.W. Chemical protein synthesis with the $\alpha$-ketoacid-hydroxylamine ligation. Acc. Chem. Res. 2017, 50, 2104-2115.

9. Baldauf, S.; Schauenburg, D.; Bode, J.W. A Threonine-Forming Oxazetidine Amino Acid for the Chemical Synthesis of Proteins through KAHA Ligation. Angew. Chem., Int. Ed. 2019, 58, 12599-12603.

10. Thompson, R. E.; Muir, T. W. Chemoenzymatic semisynthesis of proteins. Chem. Rev. 2019. 120, 3051-3126.

11. Hackeng, T.M.; Griffin, J.H.; Dawson, P.E. Protein synthesis by native chemical ligation: expanded scope by using straightforward methodology. Proc. Natl. Acad. Sci. USA, 1999, 96, 1006810073.

12. Muir, T.W.; Sondhi, D.; Cole, P.A. Expressed protein ligation: a general method for protein engineering. Proc. Natl. Acad. Sci. U. S. A. 1998, 95, 6705-6710. 
13. Conibear, A.C.; Watson, E.E.; Payne, R.J.; Becker, C.F. Native chemical ligation in protein synthesis and semi-synthesis. Chem. Soc. Rev. 2018, 47, 9046-9068.

14. Muir T. W. Semisynthesis of proteins by expressed protein ligation. Annu. Rev. Biochem. 2003, 72, 249-289.

15. Shah, N.H.; Muir, T.W. Inteins: nature's gift to protein chemists. Chem. Sci. 2014, 5, 446-461.

16. Erlanson, D.A.; Chytil, M.; Verdine, G.L. The leucine zipper domain controls the orientation of AP-1 in the NFAT AP-1 DNA complex. Chem. Biol. 1996, 3, 981-991.

17. Tolbert, T.J.; Wong, C.H. New methods for proteomic research: preparation of proteins with Nterminal cysteines for labeling and conjugation. Angew. Chem., Int. Ed. 2002, 41, 2171-2174.

18. Malins, L.R.; Mitchell, N.J.; McGowan, S.; Payne, R.J. Oxidative deselenization of selenocysteine: applications for programmed ligation at serine. Angew. Chem., Int. Ed. 2015, 127, 12907-12912.

19. Dery, S.; Reddy, P.S.; Dery, L.; Mousa, R.; Dardashti, R.N.; Metanis, N. Insights into the deselenization of selenocysteine into alanine and serine. Chem Sci, 2015, 6, 6207-6212.

20. Yan, L.Z.; Dawson, P.E. Synthesis of peptides and proteins without cysteine residues by native chemical ligation combined with desulfurization. J. Am. Chem. Soc. 2001, 123, 526-533.

21. Wang, Q.; Danishefsky, S.J. Free-radical-based, specific desulfurization of cysteine: a powerful advance in the synthesis of polypeptides and glycopolypeptides. Angew. Chem., Int. Ed. 2007, 46, 9248-9252.

22. Vamisetti, G. B.; Satish, G.; Sulkshane, P.; Mann, G.; Glickman, M. H.; Brik, A. On-demand detachment of succinimides on cysteine to facilitate (semi)synthesis of challenging proteins. J. Am. Chem. Soc. 2020, 142, 19558- 19569.

23. El Oualid, F.; Merkx, R.; Ekkebus, R.; Hameed, D.S.; Smit, J.J.; de Jong, A.; Hilkmann, H.; Sixma, T.K.; Ovaa, H. Chemical synthesis of ubiquitin, ubiquitin-based probes, and diubiquitin. Angew. Chem., Int. Ed. 2010, 49, 10149-10153.

24. Ajish Kumar, K.S.; Haj-Yahya, M.; Olschewski, D.; Lashuel, H.A.; Brik, A. Highly efficient and chemoselective peptide ubiquitylation. Angew. Chem., Int. Ed. 2009, 48, 8090-8094.

25. Yang, R.; Pasunooti, K.K.; Li, F.; Liu, X.W.; Liu, C.F. Dual native chemical ligation at lysine. J. Am. Chem. Soc. 2009, 131, 13592-13593.

26. Zhang, Y.; Xu, C.; Lam, H.Y.; Lee, C.L.; Li, X.; Protein chemical synthesis by serine and threonine ligation. Proc. Natl. Acad. Sci. USA 2013, 110, 6657-6662.

27. Liu, H., Li, X. Serine/threonine ligation: origin, mechanistic aspects, and applications. Acc. Chem. Res. 2018, 51, 1643-1655. 
28. Tan, Y.; Li, J.; Jin, K.; Liu, J.; Chen, Z.; Yang, J.; Li, X. Cysteine/Penicillamine Ligation Independent of Terminal Steric Demands for Chemical Protein Synthesis. Angew. Chem., Int. Ed. 2020, 132, 12841-12845.

29. Chow, H. Y.; Zhang, Y.; Matheson, E.; Li, X. Ligation Technologies for the Synthesis of Cyclic Peptides. Chem. Rev. 2019, 119, 9971-10001.

30. Xu, C.; Xu, J.; Liu, H.; Li, X. Development of aspartic acid ligation for peptide cyclization derived from serine/threonine ligation. Chin. Chem. Lett. 2018, 29, 1119- 1122.

31. Sun, Z.; Shang, Z.; Forelli, N.; Po, K. H. L.; Chen, S.; Brady, S. F.; Li, X. Total Synthesis of Malacidin A by $\beta$-Hydroxyaspartic Acid Ligation-Mediated Cyclization and Absolute Structure Establishment. Angew. Chem. Int. Ed. 2020, 59, 19868.

32. Chen, D.; Po, K. H. L.; Blasco, P.; Chen, S.; Li, X. Convergent Synthesis of Calcium-Dependent Antibiotic CDA3a and Analogues with Improved Antibacterial Activity via Late-Stage Serine Ligation. Org. Lett. 2020, 22, 4749-4753.

33. Li, T.; Liu, H.; Li, X. Chemical Synthesis of HMGA1a Proteins with Post-translational Modifications via Ser/Thr Ligation. Org. Lett. 2016, 18, 5944- 5947.

34. Lee, C. L.; Liu, H.; Wong, C. T. T.; Chow, H. Y.; Li, X. Enabling N-to-C Ser/Thr Ligation for Convergent Protein Synthesis via Combining Chemical Ligation Approaches. J. Am. Chem. Soc. 2016, 138, 10477- 10484

35. Lee, C. L.; Li, X. Serine/threonine ligation for the chemical synthesis of proteins. Curr. Opin. Chem. Biol. 2014, 22, 108- 114.

36. Wong, C. T. T.; Li, T.; Lam, H. Y.; Zhang, Y.; Li, X. Realizing serine/threonine ligation: scope and limitations and mechanistic implication thereof. Front. Chem. 2014, 2, 28.

37. Wong, C. T. T.; Lam, H. Y.; Song, T.; Chen, G.; Li, X. Synthesis of Constrained Head-to-Tail Cyclic Tetrapeptides by an Imine-Induced Ring-Closing/Contraction Strategy. Angew. Chem., Int. Ed. 2013, 52, 10212-10215.

38. Lam, H. Y.; Zhang, Y.; Liu, H.; Xu, J.; Wong, C. T. T.; Xu, C.; Li, X. Total Synthesis of Daptomycin by Cyclization via a Chemoselective Serine Ligation.J. Am. Chem. Soc. 2013, 135, 6272-6279.

39. Wei, T.; Liu, H.; Chu, B.; Blasco, P.; Liu, Z.; Tian, R.; Li, D.X.; Li, X. Phosphorylation-regulated HMGA1a-P53 interaction unveils the function of HMGA1a acidic tail phosphorylations via synthetic proteins. Cell Chem. Biol. 2021 28, 722-732.

40. Huang, D.L.; Montigny, C.; Zheng, Y.; Beswick, V.; Li, Y.; Cao, X.-X.; Barbot, T.; Jaxel, C.; Liang, J.; Xue, M.; Tian, C.-L.; Jamin, N.; Zheng, J.-S. Chemical Synthesis of Native S-Palmitoylated Membrane Proteins through Removable-Backbone-Modification-Assisted Ser/Thr Ligation. Angew. Chem., Int. Ed. 2020, 59, 5178- 5184. 
41. Huang, D.L.; Li, Y.; Liang, J.; Yu, L.; Xue, M.; Cao, X.-X.; Xiao, B.; Tian, C.-L.; Liu, L.; Zheng, J.-S. The New Salicylaldehyde S, S-Propanedithioacetal Ester Enables N-to-C Sequential Native Chemical Ligation and Ser/Thr Ligation for Chemical Protein Synthesis. J. Am. Chem. Soc. 2020, 142, 8790- 8799.

42. Zhang, Y.; Hirota, T.; Kuwata, K.; Oishi, S.; Gramani, S. G.; Bode, J. W. Chemical Synthesis of Atomically Tailored SUMO E2 Conjugating Enzymes for the Formation of Covalently Linked SUMO-E2-E3 Ligase Ternary Complexes. J. Am. Chem. Soc. 2019, 141, 14742- 1475.

43. Levine, P.M.; Craven, T.W.; Bonneau, R.; Kirshenbaum, K. Semisynthesis of peptoid-protein hybrids by chemical ligation at serine. Org. Lett. 2014, 16, 512-515.

44. Scaffidi, P.; Misteli, T.; Bianchi, M.E. Release of chromatin protein HMGB1 by necrotic cells triggers inflammation. Nature 2002, 418, 191-195.

45. Sims, G.P.; Rowe, D.C.; Rietdijk, S.T.; Herbst, R.; Coyle, A.J. HMGB1 and RAGE in inflammation and cancer. Annu. Rev. Immunol. 2009, 28, 367-388.

46. VanPatten, S.; Al-Abed, Y. High mobility group box-1 (HMGb1): current wisdom and advancement as a potential drug target: miniperspective. J. Med. Chem. 2017, 61, 5093-5107.

47. Arnold, K.; Xu, Y.; Sparkenbaugh, E.M.; Li, M.; Han, X.; Zhang, X.; Xia, K.; Piegore, M.; Zhang, F.; Zhang, X.; Henderson, M. Design of anti-inflammatory heparan sulfate to protect against acetaminophen-induced acute liver failure. Sci. Transl. Med. 2020, 12.

48. Stevens, N.E.; Chapman, M.J.; Fraser, C.K.; Kuchel, T.R.; Hayball, J.D.; Diener, K.R. Therapeutic targeting of HMGB1 during experimental sepsis modulates the inflammatory cytokine profile to one associated with improved clinical outcomes. Sci. Rep. 2017, 7, 1-14.

49. Lu, B.; Antoine, D.J.; Kwan, K.; Lundbäck, P.; Wähämaa, H.; Schierbeck, H.; Robinson, M.; Van Zoelen, M.A.; Yang, H.; Li, J.; Erlandsson-Harris, H. 2014. JAK/STAT1 signaling promotes HMGB1 hyperacetylation and nuclear translocation. Proc. Natl. Acad. Sci. U.S.A. 2014, 111, 3068-3073.

50. Youn, J.H.; Kwak, M.S.; Wu, J.; Kim, E.S.; Ji, Y.; Min, H.J.; Yoo, J.H.; Choi, J.E.; Cho, H.S.; Shin, J.S.; Identification of lipopolysaccharide-binding peptide regions within HMGB1 and their effects on subclinical endotoxemia in a mouse model. Eur. J. Immunol. 2011, 41, 2753-2762.

51. Deng, M.; Tang, Y.; Li, W.; Wang, X.; Zhang, R.; Zhang, X.; Zhao, X.; Liu, J.; Tang, C.; Liu, Z.; Huang, Y. 2018. The endotoxin delivery protein HMGB1 mediates caspase-11-dependent lethality in sepsis. Immunity 2018, 49, 740-753.

52. Neumann, H.; Peak-Chew, S.Y.; Chin, J.W. Genetically encoding N $\varepsilon$-acetyllysine in recombinant proteins. Nat. Chem. Biol., 2008, 4, 232-234.

53. Ito, T.; Kawahara, K.I.; Okamoto, K.; Yamada, S.; Yasuda, M.; Imaizumi, H.; Nawa, Y.; Meng, X.; Shrestha, B.; Hashiguchi, T.; Maruyama, I. Proteolytic cleavage of high mobility group box 1 
protein by thrombin-thrombomodulin complexes. Arterioscler Thromb Vasc Biol. 2008, 28, 1825 1830.

54. Gallwitz, M.; Enoksson, M.; Thorpe, M.; Hellman, L. The extended cleavage specificity of human thrombin. PloS one 2012, 7, e31756.

55. Lottenberg, R.; Hall, J.A.; Blinder, M.; Binder, E.P.; Jackson, C.M. The action of thrombin on peptide p-Nitroanilide substrates: Substrate selectivity and examination of hydrolysis under different reaction condtions. Biochim. Biophys. Acta, Protein Struct. Mol. Enzymol. 1983, 742, 539-557.

56. Chang, J.Y. Thrombin specificity: requirement for apolar amino acids adjacent to the thrombin cleavage site of polypeptide substrate. Eur. J. Biochem. 1985, 151, 217-224.

57. Rowell, J.P.; Simpson, K.L.; Stott, K.; Watson, M.; Thomas, J.O. HMGB1-facilitated p53 DNA binding occurs via HMG-Box/p53 transactivation domain interaction, regulated by the acidic tail. Structure 2012, 20, 2014-2024.

58. Unverzagt, C.; Kajihara, Y. Chemical assembly of N-glycoproteins: a refined toolbox to address a ubiquitous posttranslational modification. Chem. Soc. Rev. 2013, 42, 4408-4420.

59. Ye, F.; Zhao, J.; Xu, P.; Liu, X.; Yu, J.; Shangguan, W.; Liu, J.; Luo, X.; Li, C.; Ying, T.; Wang, J. Synthetic Homogeneous Glycoforms of the SARS-CoV-2 Spike Receptor-Binding Domain Reveals Different Binding Profiles of Monoclonal Antibodies. Angew. Chem. Int. Ed. 2021, 60, 12904-12910. 
Insert Table of Contents artwork here

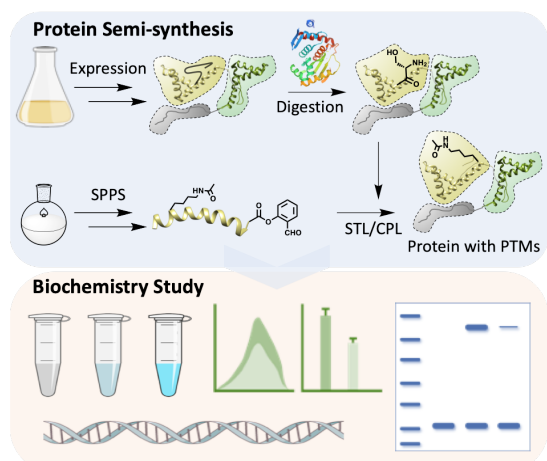

Chemical ligation mediated protein semi-synthesis has emerged as a powerful tool to produce synthetic proteins. Here, we have delved into the application of Ser/Thr ligation and Cys/Pen ligation for large protein semi-synthesis. Using this strategy, we have generated the full length tetra-acetylated HMGB1, which enabled us to illustrate that the acetylation may represent a regulatory switch to control the signal pathway and stability. 